\title{
Experiencia del Hospital Universitario de Salamanca en el tratamiento sistémico adyuvante del cáncer de mama locorregional. Resultados de un protocolo asistencial tras 7 años de seguimiento
}

E. del Barco Morillo, M. I. Ruiz Martín, J. García Mata*, C. A. Rodríguez, A. Gómez Bernal, P. Sánchez, G. Martín, E. Fonseca, Y. López Mateos**, R. García, R. Salazar, J. J. Cruz

\section{Resumen}

- Objetivos: evaluar la eficacia en términos de supervivencia libre de enfermedad (SLE) y supervivencia global (SG) y toxicidad de un protocolo asistencial para el tratamiento sistémico adyuvante del cáncer de mama.

- Material y métodos: estudio prospectivo con 308 pacientes. Se diseñó un esquema de tratamiento escalonado en relación a grupos de riesgo, factores pronósticos asociados y factores biológicos. Las pacientes recibieron 4 esquemas de tratamiento diferentes (Tamoxifeno y/o quimioterapia sin antraciclinas o uno de los dos esquemas con antraciclinas con diferente intensidad de dosis).

- Resultados: mediana de seguimiento: 7 años. La SLE y SG (士 E.E.) fue: pacientes con ganglios negativos: $86 \%( \pm 2 \%)$ y $91 \%( \pm 2 \%)$ respectivamente; pacientes con ganglios positivos: $64 \%( \pm 3)$ y $82 \%( \pm 3)$. La SLE y la SG de todo el grupo fue del $75 \%( \pm 2)$ y del $87 \%( \pm 1)$ respectivamente. La principal toxicidad encontrada fue la hematológica en las pacientes tratadas con quimioterapia.

- Conclusiones: El protocolo diseñado se ajusta a cada una de las pacientes en relación a los principales grupos de riesgo y factores pronósticos, de manera que se consigue optimizar la utilización y la selección de los diferentes agentes, tipos de quimioterapia e intensidad de dosis, evitando toxicidades innecesarias y alcanzando altos índices de eficacia en términos de SLE.

Palabras clave:

Cáncer de mama.Tratamiento adyuvante.Tamoxifeno. Quimioterapia. Antraciclinas.

Oncología, 2004; 27 (3):114-124

* Hospital Santa María Nai. Orense

** Hospital Virgen del Puerto. Plasencia

Servicio de Oncología Médica. Hospital Universitario de Salamanca 


\section{Summary}

- Purpose: To evaluate the effectiveness of the adjuvant systemic therapy of breast cancer in terms of disease-free survival (DFS), overall survival (OS) and toxicity.

- Material and methods: We made a prospective study in a cohort of 308 patients. We designed four different escalating schedule treatments in relation to risk groups, prognostic factors and biologic factors. We used tamoxifen or anthracycline alone, or tamoxifen combined with anthracycline at two different doses.

- Results: The median following up was seven years. The findings for DFS \pm s.d. and OS \pm s.d. were as follows: node-negative patients, $86 \pm 2 \%$ and $91 \pm 2 \%$, and node-positive patients, $64 \pm 3 \%$ and $82 \pm 3 \%$, respectively. For the overall group, the results were $75 \pm 2 \%$ DFS and $87 \pm 1 \%$ OS. The main toxicity was hematological in patients receiving chemotherapy.

- Conclusions: The escalating schedule treatment adjusted to patients in relation to risk group and prognostic factors optimizes the selection of chemotherapy agents and dose-intensity schedule, avoiding unnecessary toxicities and obtaining a high effectiveness in terms of DFS.

Key words: Breast cancer. Adjuvant treatment. Tamoxifen. Chemotherapy. Anthracycline

\section{Introducción}

El cáncer de mama es una de las patologías en oncología que más interés ha suscitado en los investigadores en los últimos 50 años, tanto por su incidencia y mortalidad como por sus importantes implicaciones psicosociales y sanitarias. En los países desarrollados, se estima que una de cada 8 mujeres presentará esta enfermedad y se diagnostican más de un millón de casos cada año en el mundo'. A pesar de los mejores tratamientos locorregionales, más de la tercera parte de las pacientes diagnosticadas en estadios precoces desarrollaban enfermedad recurrente o metastásica. En las pacientes con ganglios positivos, este porcentaje es del $75,5 \%$ a los 10 años, del $27,9 \%$ en las pacientes con ganglios negativos y hasta del $21 \%$ en los estadios $I^{2}$. La existencia de micrometástasis desde el momento del diagnóstico hace que ésta sea incurable con tratamientos locorregionales. Sin embargo, estas micrometástasis sí serían susceptibles de ser eliminadas con un tratamiento sistémico ${ }^{3}$.

Se han publicado importantes trabajos confirmando el beneficio del tratamiento con quimioterapia adyuvante. En 1985, el metaanálisis diseñado por el Early Breast Cancer Trialists Collaborative Group (EBCTCG), revisado y actualizado en 1990, estableció que la quimioterapia y la hormonoterapia adyuvante producen reducciones significativas de las tasas anuales de muerte y recaídas ${ }^{4,5}$. En 1998 este mismo grupo pre- sentó un nuevo metaanálisis ${ }^{6}$ y contamos además con la larga experiencia del Instituto del Cáncer de Milán con 20 años de seguimiento y del International Breast Cancer Study Group (IBCSG)7, 8 .

Los resultados de estos estudios y las conclusiones de las diferentes conferencias de consenso", establecen claramente la indicación de tratamiento con quimioterapia adyuvante en las pacientes con cáncer de mama y afectación ganglionar. En las pacientes con ganglios negativos, esta indicación continúa siendo debatida y se centra principalmente en determinar qué pacientes deben recibirlo y cuál será el esquema de tratamiento a administrar. El riesgo global de recaída en este grupo es del $20-30 \%$ a los 10 años de la intervención; por tanto, muchas de estas enfermas no se beneficiarían del tratamiento sistémico con quimioterapia. En los últimos 20 años, se han identificado diversos factores indicadores del riesgo de recaída en pacientes con ganglios linfáticos negativos (edad, el tamaño tumoral, el grado de diferenciación y el estado de los receptores hormonales) ${ }^{10,11}$. Las pacientes de riesgo moderado y alto se beneficiarían del tratamiento adyuvante con quimioterapia. Los estudios del NSABP 12 , del Intergroup (INT-0011) 13 y del grupo de Milán ${ }^{14}$, revelan el beneficio del tratamiento en términos de supervivencia libre de enfermedad (SLE) y supervivencia global (SG) sin que exista un acuerdo acerca de la composición del régimen óptimo a administrar. 


\section{E. del Barco Morillo y cols.}

En 1991 se inició en el Hospital Universitario de Salamanca un estudio prospectivo sobre el tratamiento adyuvante del cáncer de mama (Tamoxifeno y/o quimioterapia sin antraciclinas o esquemas con antraciclinas con diferente intensidad de dosis), basado en criterios de riesgo. Exponemos a continuación nuestros resultados.

\section{Material y métodos}

\section{Diseño del protocolo}

En enero de 1991 se diseña en el Servicio de Oncología Médica del Hospital Universitario de Salamanca, un protocolo asistencial para el tratamiento adyuvante de cáncer de mama locorregional con el objetivo de administrar un tratamiento escalonado que se ajustaría a cada una de las pacientes en relación al estadio, a los factores de riesgo asociados y a factores biológicos individuales de cada paciente (edad, cardiopatía, enfermedades concomitante etc, que pudieran limitar el uso de antraciclinas o de quimioterapia). Se evaluaría su eficacia mediante el cálculo de la SLE y SG por grupos de riesgo y su toxicidad, así como el efecto de la intensificación de la dosis de antraciclinas en las enfermas de alto riesgo.

Todas las pacientes fueron sometidas a cirugía con vaciamiento axilar y confirmación histológica del cáncer de mama. Se realizó un estudio de los siguientes factores pronósticos: tamaño tumoral, afectación ganglionar, estado de los receptores hormonales $(\mathrm{RH})$ y grado histológico.

La valoración inicial previa al tratamiento consistió en: historia clínica y examen físico completo, hemograma y bioquímica completa (con pruebas de función hepática, renal y electrolitos) marcadores tumorales (Antígeno Polipéptido Tisular -TPA-, CA 15-3), radiografía de tórax, ecografía abdominal, gammagrafía ósea, mamografía contralateral y electrocardiograma, que debían ser normales. Se determinó la fracción de eyección ventricular (FE) en aquellas pacientes que iban a recibir tratamiento con antraciclinas.

\section{Esquemas de tratamiento}

\section{- Hormonoterapia:}

Tamoxifeno: $20 \mathrm{mg} /$ día durante 5 años en todas las pacientes con RH positivos. En las pacientes que recibieron quimioterapia, el tratamiento hormonal se administró de manera secuencial.

\section{- Quimioterapia:}

CMF: ciclofosfamida $600 \mathrm{mg} / \mathrm{m}^{2}$ iv día 1 , metotrexate $40 \mathrm{mg} / \mathrm{m}^{2}$ iv día 1 , 5fluoracilo $600 \mathrm{mg} / \mathrm{m}^{2}$ iv día 1, cada 3 semanas, por 6 ciclos.

CAF: ciclofosfamida $500 \mathrm{mg} / \mathrm{m}^{2}$ iv día 1 , adriamicina $50 \mathrm{mg} / \mathrm{m}^{2}$ iv día 1 , 5fluoracilo $500 \mathrm{mg} / \mathrm{m}^{2}$ iv día 1, cada3 semanas, por 6 ciclos.

SCAF: ciclofosfamida $600 \mathrm{mg} / \mathrm{m}^{2}$ iv día 1, adriamicina $60 \mathrm{mg} / \mathrm{m}^{2}$ iv día 1, 5fluoracilo $600 \mathrm{mg} / \mathrm{m}^{2}$ iv día 1, cada 3 semanas, por 6 ciclos.

Se programó que la quimioterapia adyuvante se iniciaría dentro de las 5 semanas siguientes a la cirugía.

\section{- Radioterapia:}

Se administró una vez terminado el tratamiento quimioterápico: en todas las pacientes en las que se realizó tratamiento quirúrgico conservador "sobre la glándula" y en aquéllas con 4 o más ganglios axilares positivos, N2 ○ T>5 cm. sobre el lecho de mastectomía y regiones ganglionares.

\section{Elección del tratamiento según grupos pronósticos}

Una vez conocido el estadio y valorados factores pronósticos, se estratificaba a las pacientes y se establecía el tratamiento correspondiente de la siguiente manera:

\section{Pacientes con ganglios negativos}

Se estratificaron por grupos de riesgo basados en los criterios establecidos en las diferentes Conferencias de Consenso del National Institutes of Health $(\mathrm{NIH})$ y Conferencias de Sant Gallen ${ }^{15,16 .}$

- Tumores menores o igual a 2 centímetros (T1): se consideraron de bajo riesgo las pacientes que cumplían todos y cada uno de los siguientes criterios: postmenopáusica, receptores hormonales positivos y grado I de diferenciación. Este grupo de pacientes recibió tratamiento con hormonoterapia. El resto de las pacientes de este subgrupo que no cumplieron todos estos criterios, fueron consideradas de alto riesgo y recibieron tratamiento con quimioterapia según protocolo $C M F$.

- Tumores mayores de 2 centímetros (>T1): se consideraron de alto riesgo y fueron tratadas con quimioterapia según protocolo CAF.

\section{Pacientes con ganglios positivos}

- 1-3 ganglios positivos: recibieron quimioterapia según protocolo $C A F$. 
- 4 -9 ganglios positivos: recibieron quimioterapia según protocolo $S C A F$.

- Más de 9 ganglios positivos y N2: recibieron quimioterapia según protocolo SCAF.

En las pacientes mayores de 65 años y dependiendo de su estado general, se consideró un escalón de tratamiento inferior: se aplicó el esquema correspondiente al grupo de riesgo inmediatamente inferior. En aquellas mayores de 75 años, el tratamiento fue individualizado: ninguna recibió tratamiento con antraciclinas y sólo se administró quimioterapia con CMF si tenían buen estado general (Índice de Karnofsky $\geq 70$ ) y no presentaban enfermedades concomitantes graves. Se realizó una ventriculografía isotópica antes de iniciar un tratamiento programado con antraciclinas y si la $\mathrm{FE}$ basal era inferior al $45 \%$ se administraba CMF. $\mathrm{Si}$ la $\mathrm{FE}$, durante un tratamiento con antraciclinas disminuía un $10 \%$ del valor basal, se suspendía y se continuaba con CMF.

Para la evaluación de la toxicidad se siguieron los criterios de la Organización Mundial de la Salud (OMS). Antes de cada ciclo de quimioterapia, se requería un recuento completo de células sanguíneas. Se retrasaba una semana la administración de quimioterapia si: recuento de ganulocitos inferior a $100.000 \mathrm{x}$ $10^{9}$ células $/ \mathrm{I}$ y recuento de plaquetas inferior a 1.500 $\times 10^{9}$ células/l. Si no se producía recuperación hematológica se reducían un $25 \%$ las dosis de todos los fármacos. Si en el día 1 de una tanda programada de quimioterapia, los recuentos de granulocitos eran inferiores a $1.000 \times 10^{9}$ células/I o de plaquetas inferiores a $75.000 \times 10^{9}$ células/l, se reducían las dosis de todos los fármacos en un $25 \%$ y se administraba el tratamiento cuando se producía la recuperación hematológica.

\section{Seguimiento}

Una vez finalizado el tratamiento, se programó para todas las pacientes un calendario de revisiones clínicas y pruebas complementarias trimestrales durante los dos primeros años, cada 6 meses hasta los 5 años y posteriormente, anuales. Se realizó exploración físi$\mathrm{ca}$, analítica general (hemograma y bioquímica), marcadores tumorales (TPA y CA15-3), radiografía de tórax, ecografía hepática y gammagrafía ósea al final de tratamiento quimioterápico. Posteriormente, en todas las revisiones: exploración física, analítica general (hemograma y bioquímica), marcadores tumorales (TPA y CA 15-3) y radiografía de tórax. Mamografía y gammagrafía ósea anual; cada 6 meses hasta los 2 años ecografía hepática y después anual. Además, se realizaron revisiones ginecológicas periódicas en aquellas pacientes con tratamiento hormonal.

Se consideró fracaso del tratamiento adyuvante, la evidencia de manifestaciones de enfermedad documentada mediante exámenes clínicos, radiológicos o de laboratorio, y cuando era posible, histológicos. Se incluyó como fracaso del tratamiento la aparición de cáncer de mama contralateral o en la misma mama en las pacientes tratadas con cirugía conservadora.

\section{Análisis estadístico}

Mediante el método de Kaplan-Meier ${ }^{17}$ para el cálculo de las probabilidades de supervivencia, en cualquier condición, se estimó la supervivencia libre de enfermedad (SLE) en meses desde el momento de la intervención hasta la última fecha de seguimiento o la aparición de la primera recaída o metástasis a distancia, y la supervivencia global (SG) en meses, desde el momento de la intervención hasta la última fecha de seguimiento o la fecha de la muerte por cualquier causa. Las diferencias en las distribuciones de tiempo fueron evaluadas utilizando el test de log-rank ${ }^{18}$.

La intensidad de dosis administrada (IDA) en el caso de esquema quimioterápico con SCAF, se definió como la cantidad total (en $\mathrm{mg}$ ) de fármaco administrado durante el tratamiento, dividido por la superficie corporal total de la paciente y por el número total de semanas de tratamiento recibido (en $\mathrm{mg} / \mathrm{m}^{2} /$ semana) ${ }^{19}$.

\section{Resultados}

Desde enero de 1991 hasta noviembre de 1998 fueron incluidas en este protocolo un total de 308 pacientes. En la Tabla I se muestra la distribución de las pacientes por grupos de riesgo y el tratamiento administrado. El 50,3\% fueron pacientes con ganglios negativos. Las características de las pacientes se resumen en la Tabla II. La mediana de edad fue de 60 años y casi el $75 \%$ fueron pacientes postmenopáusicas. La histología más frecuente fue carcinoma ductal infiltrante $(89 \%)$. La información de seguimiento se actualizó hasta marzo de 2003. El tiempo mínimo de seguimiento en la evaluación actual es de 5 años en el $95 \%$ de las pacientes. La mediana de seguimiento es de 7 años (intervalo 1 año-12,2 años). En relación al esquema terapéutico con SCAF, la intensidad de dosis relativa (IDR) respecto a la dosis óptima fue mayor del $95 \%$ en el $53 \%$ de las pacientes; entre el 90 y el $95 \%$ de IDR se administró en el $30 \%$ y menos del $90 \%$ de IDR en tan sólo el 17\% de las pacientes. La SLE (Fig. 1) y SG (Fig. 2) ( \pm E.E.) a los 5 años de las pacientes con ganglios 


\section{E. del Barco Morillo y cols.}

TABLA 1

Distribución de las pacientes por grupos de riesgo y tratamiento administrado

\begin{tabular}{lccccc}
\hline Ganglios negativos & TAM & CMF & CAF & SCAF & Total \\
\hline T1 & 35 & 27 & 0 & 0 & 62 \\
$\geq$ T2 & 15 & 37 & 41 & 0 & 93 \\
Ganglios positivos & & & & & \\
\hline N1-3 & 12 & 25 & 38 & 0 & 75 \\
N4-9 & 1 & 13 & 14 & 27 & 55 \\
N $\geq 10$ Y N2 & 5 & 5 & 4 & 9 & 23 \\
& & & & Total & 308 \\
\hline
\end{tabular}

TAM: tamoxifeno.

CMF: ciclofosfamida 600 - metotrexate 40 - 5fluoracilo $600\left(\mathrm{mg} / \mathrm{m}^{2}\right)$.

CAF: ciclofosfamida 500 - adriamicina 50 - 5fluoracilo $500\left(\mathrm{mg} / \mathrm{m}^{2}\right)$.

SCAF: ciclofosfamida 600 - adriamicina 60 - 5 fluoracilo $600\left(\mathrm{mg} / \mathrm{m}^{2}\right)$.

TABLA \|

\section{Características de las pacientes}

\begin{tabular}{lrr} 
№ de pacientes & 308 & \\
\hline Edad (mediana y rango) & 60 & $(29-84)$ \\
Postmenopáusicas & 231 & $(74.5 \%)$ \\
R. Estrógenos + & 125 & $(40.32 \%)$ \\
R. Progesterona + & 160 & $(51.6 \%)$ \\
Histología & & \\
Ductal & & \\
Lobulillar & 274 & $(89 \%)$ \\
Mucinoso & 12 & $(3.8 \%)$ \\
Medular & 10 & $(3.2 \%)$ \\
Papilar & 6 & $(1.9 \%)$ \\
Tubular & 4 & $(1.29 \%)$ \\
& 2 & $(0.64 \%)$
\end{tabular}

\section{Grado histológico}

\begin{tabular}{lrr} 
III & 47 & $(15.16 \%)$ \\
III & 136 & $(44.19 \%)$ \\
Ganglios negativos & 125 & $(40.64 \%)$ \\
Tla & 155 & $(50.3 \%)$ \\
Tlb & 1 & \\
Tlc & 10 & \\
T2 o Mayor & 51 & \\
Ganglios positivos & 93 & \\
De 1 a 3 & 153 & $(49.67 \%)$ \\
De 4 a 9 & 75 & \\
> 10 0 N2 & 55 & \\
\hline
\end{tabular}

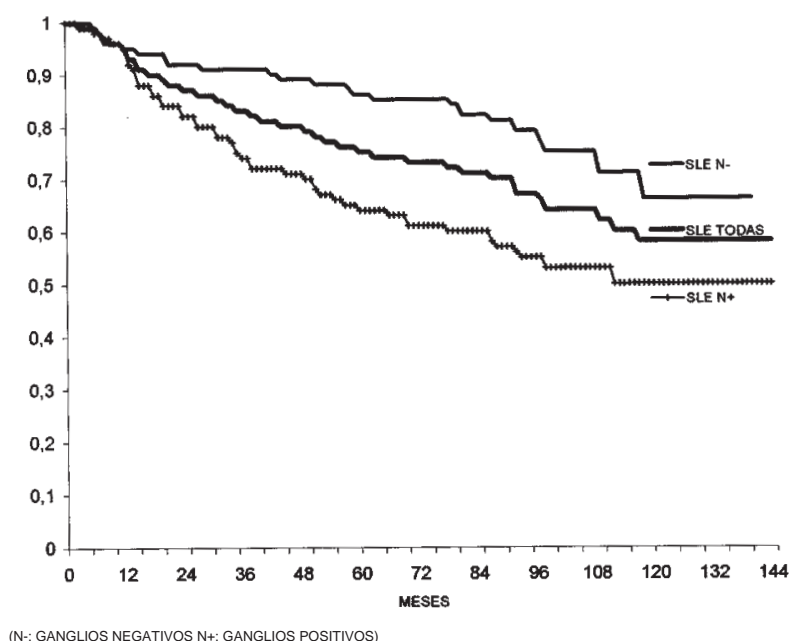

Fig. 1. Supervivencia libre de enfermedad (SLE).

negativos fue del $86 \%( \pm 2 \%)$ y del $91 \%( \pm 2 \%)$ respectivamente y de las pacientes con ganglios positivos del $64 \%( \pm 3)$ y del $82 \%( \pm 3)$. La SLE y la SG de todo el grupo fue del $75 \%( \pm 2)$ y del $87 \%( \pm 1)$. En las Figuras 3 y 4 se muestra gráficamente la SLE y la SG de las pacientes estratificadas por grupos de riesgo y en la Tabla III los datos correspondientes.

Se han producido 93 eventos y 62 éxitus (Tabla III). La mediana de tiempo en meses hasta la recidiva en las pacientes con 10 o más ganglios afectados y N2 es de 31 meses. En los demás grupos no se ha alcanzado. La localización de las metástasis fue: dérmica, 21 pacientes; ganglionar, 5; hepática, 12; pleural, 3; pul- 


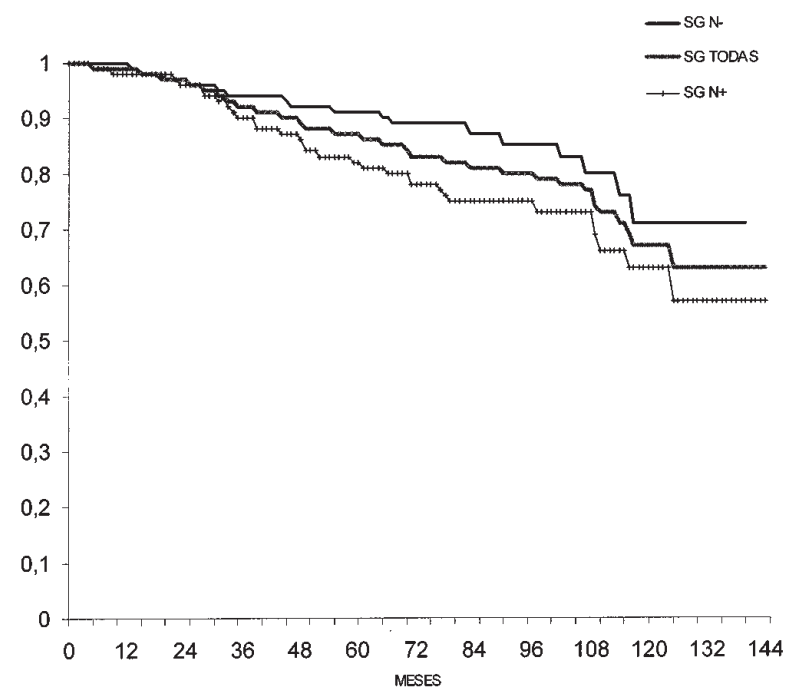

(N-: GANGLIOSL NEGATIVOS N+: GANGLIOS POSITIVOS)

Fig. 2. Supervivencia global (SG).

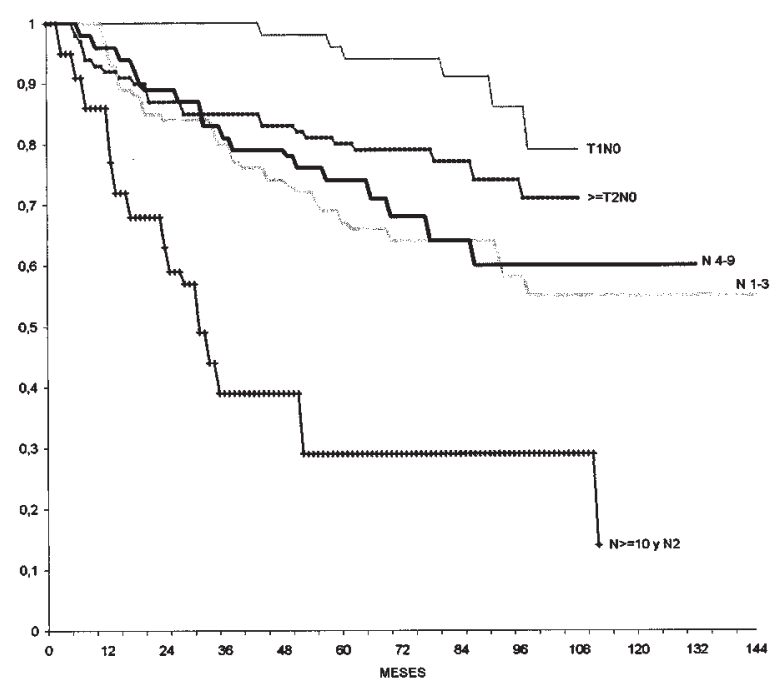

Fig. 3. Supervivencia libre de enfermedad (SLE) por subgrupos pronósticos.

monar, 13; cerebral, 10; mama contralateral, 15; partes blandas, 1 paciente. 240 pacientes recibieron tratamiento con quimioterapia. La principal toxicidad encontrada fue gastrointestinal y hematológica y se resume en la Tabla IV. Los efectos adversos en el grupo de pacientes tratadas con SCAF fueron más evidentes, siendo necesaria la reducción de la dosis de antraciclinas en dos pacientes por disminución de la FE durante el tratamiento. En este grupo de pacientes se retrasa-

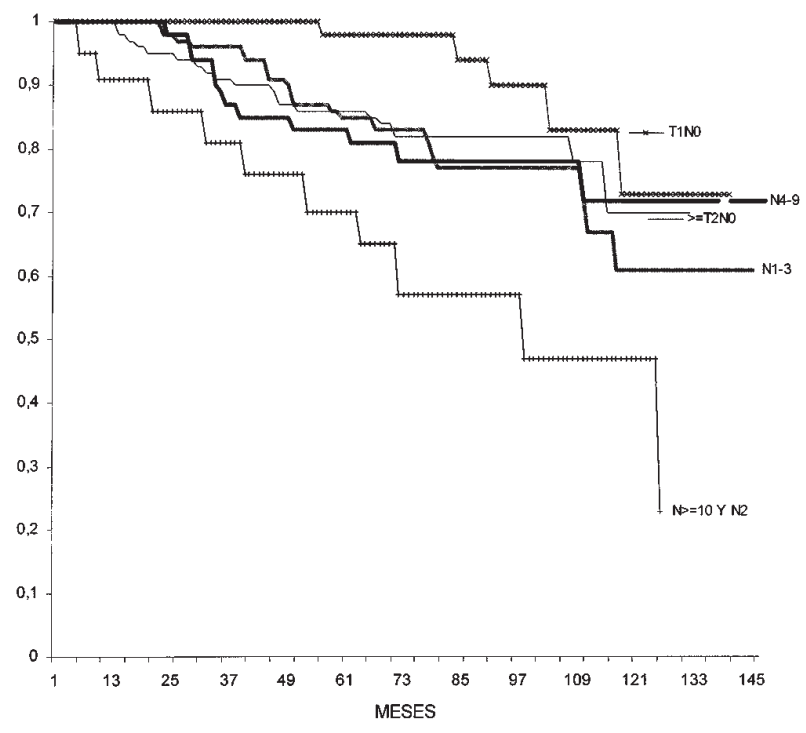

Fig. 4. Supervivencia global (SG) por subgrupos pronósticos.

ron 29 ciclos, siendo la neutropenia la causa del retraso en el $75 \%$ de ellos; en una paciente se suspendió el tratamiento por arritmia cardíaca y otra falleció a causa de una insuficiencia cardíaca congestiva durante el tratamiento. Hubo dos casos de neutropenia febril, ambos en el grupo tratado con SCAF. Se han observado 10 segundos tumores (dos en vejiga, un cáncer epidermoide de pulmón, uno de recto, uno de cérvix, dos en el sistema nervioso central y tres endometriales). Los tres cánceres endometriales afectaron a mujeres tratadas con Tamoxifeno (1,4\% de 209 pacientes).

\section{Discusión}

Los beneficios aportados por el tratamiento adyuvante del cáncer de mama precoz es un hecho ya demostrado 4,5 . El tratamiento hormonal adyuvante con Tamoxifeno se recomienda durante 5 años a todas las mujeres que expresan receptores hormonales ${ }^{5,20}$, no estando indicado en pacientes con receptores hormonales negativos ${ }^{21}$. Su uso ha sido asociado con ciertos efectos tóxicos: desarrollo de cáncer endometrial ${ }^{22}$, trombosis de venas profundas y embolia pulmonar con una incidencia que varía entre el $1 \%$ al $2 \%^{21}$. Los estudios que comparan la combinaciones de Tamoxifeno y quimioterapia administradas concurrentemente han demostrados aumentar los efectos tóxicos con resultados diversos sobre la SLE y SG $23-25$. En nuestro protocolo, 209 pacientes han recibido tratamiento hormonal con Tamoxifeno ( $20 \mathrm{mg} /$ día durante 5 años) y 


\section{E. del Barco Morillo y cols.}

TABLA III

Eventos y éxitus. Supervivencia libre de enfermedad (SLE) y Supervivencia global (SG) a los 5 años

\begin{tabular}{|c|c|c|c|c|c|c|}
\hline & & TAM & CMF & CAF & SCAF & Total \\
\hline Total & $\begin{array}{l}\text { Total pacientes } \\
\text { Eventos } \\
\text { Exitus } \\
\text { SLE (EE) } \\
\text { SG (EE) }\end{array}$ & $\begin{array}{r}68 \\
16(23.5 \%) \\
13(19 \%)\end{array}$ & $\begin{array}{r}107 \\
30(28 \%) \\
22(20 \%)\end{array}$ & $\begin{array}{r}97 \\
36(37 \%) \\
20(20.5 \%)\end{array}$ & $\begin{array}{r}36 \\
11(30 \%) \\
7(19.4 \%)\end{array}$ & $\begin{array}{r}308 \\
93(30 \%) \\
62(20 \%) \\
75 \%( \pm 2 \%) \\
87 \%( \pm 1 \%)\end{array}$ \\
\hline NO & $\begin{array}{l}\text { Total pacientes } \\
\text { Eventos } \\
\text { Exitus } \\
\text { SLE (EE) } \\
\text { SG (EE) }\end{array}$ & $\begin{array}{r}50 \\
7(14 \%) \\
6(12 \%)\end{array}$ & $\begin{array}{r}64 \\
14(21.8 \%) \\
11(17 \%)\end{array}$ & $\begin{array}{r}41 \\
9(21.9 \%) \\
5(12 \%)\end{array}$ & & $\begin{array}{r}155 \\
30(19.3 \%) \\
22(14 \%) \\
86 \%( \pm 2 \%) \\
91 \%( \pm 2 \%)\end{array}$ \\
\hline T1 & $\begin{array}{l}\text { Total pacientes } \\
\text { Eventos } \\
\text { Exitus } \\
\text { SLE (EE) } \\
\text { SG (EE) }\end{array}$ & $\begin{array}{r}35 \\
4(11.4 \%) \\
3(8.5 \%)\end{array}$ & $\begin{array}{r}27 \\
4(14.8 \%) \\
2(7.4 \%)\end{array}$ & & & $\begin{array}{r}62 \\
8(12.9 \%) \\
5(8.06 \%) \\
96 \%( \pm 2 \%) \\
98 \%( \pm 1 \%)\end{array}$ \\
\hline$\geq \mathrm{T} 2$ & $\begin{array}{l}\text { Total pacientes } \\
\text { Eventos } \\
\text { Exitus } \\
\text { SLE (EE) } \\
\text { SG (EE) }\end{array}$ & $\begin{array}{r}15 \\
3(20 \%) \\
3(20 \%)\end{array}$ & $\begin{array}{r}37 \\
10(27 \%) \\
9(24.3 \%)\end{array}$ & $\begin{array}{r}41 \\
9(21.9 \%) \\
5(12.1 \%)\end{array}$ & & $\begin{array}{r}93 \\
22(23.6 \%) \\
17(18.2 \%) \\
80 \%( \pm 4 \%) \\
86 \%( \pm 3 \%)\end{array}$ \\
\hline $\mathrm{N}+$ & $\begin{array}{l}\text { Total pacientes } \\
\text { Eventos } \\
\text { Exitus } \\
\text { SLE (EE) } \\
\text { SG (EE) }\end{array}$ & $\begin{array}{r}18 \\
9(50 \%) \\
7(38.8 \%)\end{array}$ & $\begin{array}{r}43 \\
16(37.2 \%) \\
11(25.5 \%)\end{array}$ & $\begin{array}{r}56 \\
27(48.2 \%) \\
15(26.7 \%)\end{array}$ & $\begin{array}{r}36 \\
11(30.5 \%) \\
7(19.4 \%)\end{array}$ & $\begin{array}{r}153 \\
63(41.1 \%) \\
40(26.1 \%) \\
64 \%( \pm 3 \%) \\
82 \%( \pm 3 \%)\end{array}$ \\
\hline N1-3 & $\begin{array}{l}\text { Total pacientes } \\
\text { Eventos } \\
\text { Exitus } \\
\text { SLE (EE) } \\
\text { SG (EE) }\end{array}$ & $\begin{array}{r}12 \\
5(41.6 \%) \\
4(33.3 \%)\end{array}$ & $\begin{array}{r}25 \\
8(32 \%) \\
6(24 \%)\end{array}$ & $\begin{array}{r}38 \\
16(42.1 \%) \\
8(21 \%)\end{array}$ & & $\begin{array}{r}75 \\
29(38.6 \%) \\
18(24 \%) \\
67 \%( \pm 5 \%) \\
85 \%( \pm 4 \%)\end{array}$ \\
\hline N4-9 & $\begin{array}{l}\text { Total pacientes } \\
\text { Eventos } \\
\text { Exitus } \\
\text { SLE (EE) } \\
\text { SG (EE) }\end{array}$ & $\begin{array}{r}1 \\
1(100 \%) \\
1(100 \%)\end{array}$ & $\begin{array}{r}13 \\
4(30.7 \%) \\
2(15.3 \%)\end{array}$ & $\begin{array}{r}14 \\
7(50 \%) \\
5(35.7 \%)\end{array}$ & $\begin{array}{r}27 \\
6(22.2 \%) \\
4(14.8 \%)\end{array}$ & $\begin{array}{r}55 \\
18(32.7 \%) \\
12(21.8 \%) \\
74 \%( \pm 6 \%) \\
83 \%( \pm 5 \%)\end{array}$ \\
\hline $\mathrm{N} \geq 10$ y $\mathrm{N} 2$ & $\begin{array}{l}\text { Total pacientes } \\
\text { Eventos } \\
\text { Exitus } \\
\text { SLE (EE) } \\
\text { SG (EE) }\end{array}$ & $\begin{array}{r}5 \\
3(60 \%) \\
2(40 \%)\end{array}$ & $\begin{array}{r}5 \\
4(80 \%) \\
3(60 \%)\end{array}$ & $\begin{array}{r}4 \\
4(100 \%) \\
2(50 \%)\end{array}$ & $\begin{array}{r}9 \\
5(55.5 \%) \\
3(33.3 \%)\end{array}$ & $\begin{array}{r}23 \\
16(69.5 \%) \\
10(43.4 \%) \\
29 \%( \pm 12 \%) \\
70 \%( \pm 10 \%)\end{array}$ \\
\hline
\end{tabular}

EE: Error estándar. TAM: tamoxifeno. CMF: ciclofosfamida 600 - metotrexate 40 - 5 fluoracilo $600\left(\mathrm{mg} / \mathrm{m}^{2}\right)$. CAF: ciclofosfamida 500 - adriamicina 50 - 5fluoracilo $500\left(\mathrm{mg} / \mathrm{m}^{2}\right)$.

SCAF: ciclofosfamida 600 - adriamicina 60 - 5fluoracilo $600\left(\mathrm{mg} / \mathrm{m}^{2}\right)$. 


\section{Principal toxicidad (OMS) encontrada en las pacientes que recibieron quimioterapia, por tipo de quimioterapia y} ciclos administrados (\%)

\begin{tabular}{|c|c|c|c|c|c|c|c|c|c|c|c|c|}
\hline & \multicolumn{3}{|c|}{ Grado I } & \multicolumn{3}{|c|}{ Grado II } & \multicolumn{3}{|c|}{ Grado III } & \multicolumn{3}{|c|}{ Grado IV } \\
\hline Náuseas/vómitos & 5 & 6 & 5.5 & 3 & 5 & 6.7 & 0 & 3 & 4.2 & 0 & 0 & 0 \\
\hline Diarrea & 0.6 & 0 & 0 & 0 & 0.4 & 0 & 0 & 1 & 0 & 0 & 0 & 0 \\
\hline Mucositis & 0 & 1 & 0.4 & 0.4 & 1 & 1.2 & 0 & 0.5 & 1.2 & 0 & 0 & 0 \\
\hline Anemia & 0.8 & 1 & 4.6 & 0 & 2 & 0 & 0 & 0 & 0 & 0 & 0 & 0 \\
\hline Leucopenia & 10 & 11.5 & 10.5 & 5 & 1 & 0.8 & 0 & 0 & 0.8 & 0 & 0 & 0 \\
\hline Neutropenia & 10 & 11.5 & 7.2 & 10 & 6 & 6.7 & 3 & 3 & 2.5 & 0 & 0 & 1 \\
\hline
\end{tabular}

141 de ellas recibieron quimioterapia y hormonoterapia de manera secuencial. No se ha detectado ningún caso de trombosis venosa profunda ni embolia pulmonar. Tres pacientes desarrollaron cáncer endometrial $(1,4 \%)$. Todas ellas eran postmenopáusicas: una, con 71 años en el momento del diagnóstico, falleció a los 9 años y medio por recidiva pleural y ósea; otra de 63 años desarrolló un carcinoma endometrial de bajo grado y continúa viva y libre de enfermedad a los 7 años del diagnóstico y la última, de 71 años, desarrolló también un cáncer de mama contralateral. En la actualidad continúa viva y libre de enfermedad a los 7 años del diagnóstico. También se ha relacionado al Tamoxifeno con aumento en el riesgo de derrames cerebrales $^{26}$. Dos pacientes en nuestro estudio fallecieron por accidente cerebrovascular, pero ninguna estaba en tratamiento con Tamoxifeno.

El grado de afectación axilar es el factor pronóstico independiente que mejor predice la evolución de las enfermas ${ }^{27}$. En las pacientes sin afectación axilar la decisión del tipo de tratamiento a administrar se basa en la edad, tamaño tumoral, estado de los receptores hormonales y grado histológico9-11, 28. En nuestro estudio, las enfermas sin afectación axilar fueron subclasificadas siguiendo las directrices de las diferentes Conferencias de Consenso. Se administró CMF (las 3 drogas intravenosas, el día 1, cada 3 semanas) en aquellas pacientes con tumores de dos centímetros o menos y que eran consideradas de alto riesgo (premenopáusica, o RH negativos o grado II-III). A pesar de que el esquema CMF desarrollado por el Instituto del Cáncer de Milán consigue una mayor intensidad de dosis, la administración intravenosa en el día 1, cada tres semanas, ha demostrado ser equivalente al CMF clásico y consigue un mayor cumplimiento por parte de las pacientes y menor toxicidad ${ }^{29}$, razón por la cual éste fue elegido en nuestro protocolo para el tratamiento adyuvante. 35 pacientes con tumores $\leq 2 \mathrm{~cm}$. de bajo riesgo recibieron exclusivamente tratamiento hormonal y 64 recibieron el esquema con CMF. La SLE y la SG a los 5 años de las pacientes sin afectación axilar, fue del $96 \%( \pm 2 \%$ ) y del $98 \%$ ( $\pm 1 \%$ ). La toxicidad de este esquema quimioterápico es bien tolerada, predominando la leucopenia y la toxicidad gastrointestinal grado I-II. Hasta ahora no se han detectado segundos tumores en relación al tratamiento administrado. Al hacer el estudio de supervivencia por subgrupos pronósticos, el número de pacientes en cada grupo se reduce considerablemente, pero aún así consideramos estos resultados como excelentes.

El tratamiento con antraciclinas en pacientes con ganglios negativos es algo más controvertido en la actualidad. El metaanálisis del EBCTCG analizó 11 ensayos en los cuales las mujeres se dividieron en grupos: unas, recibieron tratamiento con regímenes a base de antraciclinas y otras recibieron CMF solamente. A los 5 años, las diferencias en la ausencia de recaídas y la supervivencia favorecieron a los regímenes a base de antraciclinas $^{4}$. En el estudio de Hutchins et al. (Estudio 0102 del U.S. Intergroup), se realizó la mayor comparación directa del CAF con el CMF en pacientes con cáncer de mama sin afectación axilar. A los 5 años, el CAF fue discretamente superior al CMF tanto en la SLE como en la $\mathrm{SG}^{30}$. En nuestro estudio, 93 pacientes con ganglios negativos fueron consideradas de alto riesgo por presentar tumores de más de $2 \mathrm{~cm}(44,5 \%$ con tumores de más de $3 \mathrm{~cm}$ ), pero además la gran mayoría de estas pacientes presentaban otros factores de mal pronóstico: $70 \%$ fueron tumores de bajo grado de diferenciación y el $60 \%$ fueron aneuploides. 41 pacientes fueron tratadas con antraciclinas y el resto con CMF y/o tratamiento hormonal siguiendo el diseño de nuestro protocolo. La SLE y la SG a los 5 años fue del $80 \%( \pm 4 \%$ ) y del $86 \%( \pm 3 \%)$. El riesgo de toxicidad 


\section{E. del Barco Morillo y cols.}

cardíaca asociada a los regímenes que contienen antraciclinas, es mínimo con las dosis utilizadas, especialmente si se administra en infusión continua de 4872 horas $^{31}$. Otra de las complicaciones con antraciclinas a largo plazo, del tratamiento es el desarrollo de leucemia mieloide aguda sobre todo si se asocia a tratamiento con radioterapia ${ }^{32}$. En nuestro estudio la adriamicina, se administraba en bolo y no se ha evidenciado toxicidad cardíaca en las pacientes, ni hasta el momento, algún caso de leucemia.

En las pacientes con ganglios axilares positivos, los dos esquemas de tratamiento administrados en nuestro protocolo se basaron en antraciclinas. Su uso produce un beneficio en la supervivencia comparado con los regímenes basados en $\mathrm{CMF}$, con una mejoría en el riesgo anual de recurrencia y de muerte del $11 \%$ y $12 \%$, respectivamente ${ }^{4,33}, 34$. De las 75 pacientes con afectación de 1 a 3 ganglios, 38 recibieron quimioterapia con dosis estándar de antraciclinas y el resto quimioterapia con CMF o sólo hormonoterapia siguiendo el esquema de nuestro protocolo: eran pacientes que no podían recibir antraciclinas (FE baja y/o mayores de 65 años) o quimioterapia con CMF (pacientes mayores de 75 años y/o con enfermedades concomitantes que limitaban el uso de ésta). No se observaron diferencias significativas entre los subgrupos de tratamiento, ni en la SLE ni en la SG. Aunque el número limitado de pacientes no nos permite sacar conclusiones definitivas, cabría pensar que en aquellas pacientes con baja afectación axilar (1-3 ganglios) el tratamiento adyuvante con CMF y/o hormonal podría ser una opción válida cuando no se pueden administrar regímenes con antraciclinas.

Importantes estudios sugieren la existencia de una relación dosis-respuesta en el tratamiento adyuvante ${ }^{35}$, de manera que niveles de dosis por debajo del estándar producirían claramente peores resultados ${ }^{36,37}$. Sin embargo, varios ensayos clínicos han probado la quimioterapia con mayor intensidad de dosis y sus resultados no han indicado beneficios ${ }^{38-40}$. El esquema con SCAF $1600 / 60 / 600 \mathrm{mg} / \mathrm{m}^{2}$ de CAF cada 3 semanas durante 6 ciclos) consigue un aumento importante en la intensidad de dosis respecto al CAF clásico. Los resultados obtenidos en el grupo de pacientes con más de 9 ganglios y N2 resultan poco concluyentes, ya que se trata de un número muy escaso de pacientes. La mayoría de las mujeres diagnosticadas de cáncer de mama con este grado de afectación, durante el período que se puso en marcha el protocolo fueron incluidas en tratamientos de intensificación con soporte de células sten periféricas. El resto (23 enfermas) fueron tratadas con el protocolo asistencial descrito y conforman un grupo muy heterogéneo de pacientes con eda- des superiores a 60 años, peor estado general y con problemas cardíacos alguna de ellas. A pesar de ello, podemos concluir que se trata de un grupo de mujeres con muy mal pronóstico (mediana de tiempo hasta la aparición de la recaída de 27 meses).

En los resultados de nuestro estudio, la SLE y la SG los 5 años de las pacientes con 1-3 ganglios es similar a las de $4-9$ ganglios $167 \%$ vs $74 \%$ y $85 \%$ vs $83 \%$ respectivamente). Este hecho nos resulta destacable, ya que estas últimas tienen a priori un peor pronóstico. Cabría pensar que la intensidad de dosis del SCAF podría tener un efecto beneficioso sobre el grupo de enfermas de peor pronóstico. Sería necesario un mayor seguimiento en el análisis, que nos permitiera realizar conclusiones más exactas acerca de dicho efecto. La toxicidad del SCAF fue más importante que la del CAF clásico, aunque resultó tolerable, destacando la toxicidad cardiológica. La forma de administración del esquema no impidió que se alcance la dosis óptima calculada ya que tan sólo el $17 \%$ de las pacientes alcanzaron menos del $90 \%$ de intensidad de dosis relativa respecto a la dosis óptima calculada.

En conclusión, tras una mediana de seguimiento de 7 años, el protocolo diseñado alcanza un alto índice de eficacia en términos de SLE, aunque son necesarios algunos años más de seguimiento para confirmar estos resultados a largo plazo. También es necesario un mayor seguimiento para evaluar y confirmar los resultados en la SG. Este protocolo escalonado, se ajusta a cada una de las pacientes en relación al estadio de la enfermedad, a los principales factores de riesgo asociados y a factores biológicos individuales que limitan el uso de antraciclinas o de quimioterapia. Consigue optimizar la utilización de los diferentes fármacos, tipos de quimioterapia e intensidad de dosis, evitando toxicidades innecesarias sin perder con ello efectividad. Todavía se precisan estudios sobre nuevos factores pronósticos y de respuesta que nos permitan establecer más claramente qué pacientes sin afectación axilar no se van a beneficiar de un tratamiento con quimioterapia complementaria. En aquellas pacientes que precisan tratamiento quimioterápico, el esquema con CMF continúa siendo una opción válida, sobre todo cuando existen limitaciones para la utilización de antraciclinas: más del $30 \%$ de las 246 pacientes programadas para antraciclinas recibieron $\mathrm{CMF}$, sin que ello supusiera un deterioro en la efectividad (la SLE a los 5 años en las pacientes con ganglios negativos fue del $86 \%$ ). Las pacientes con ganglios positivos tienen un pronóstico infausto a pesar de los tratamientos adyuvantes administrados (SLE del $64 \%$ a los 5 años, con una mediana de tiempo hasta la recaída de 31 meses en las pacientes de peor pronóstico). La mayor intensi- 
dad de dosis conseguida con el SCAF podría tener un efecto beneficioso sobre el grupo de enfermas de mal pronóstico (4-9 ganglios afectados) con una SLE a los 5 años del $74 \%$, aunque es necesario un mayor seguimiento en el análisis que nos permitiera realizar conclusiones más exactas acerca dicho efecto.

\author{
Correspondencia: \\ Dr. E. del Barco Morillo \\ Servicio de Oncología Médica \\ Hospital Universitario de Salamanca \\ Paseo de San Vicente, 58-182 \\ E-37007 Salamanca \\ oncologia@oncologiasalamanca.org
}

\section{Bibliografía}

1. Cancer Incidence, Mortality and Prevalence Worldwide (2000 estimates). Disponible en: http://wwwdep.iarc.fr/globocan/globocan.html.

2. Valagussa P, Bonadonna $G$ and Veronesi U. Patterns of relapse and survival following radical mastectomy. Cancer 1978; 41:1170-78.

3. Goldie J. Scientific basis for adyuvant and primary (neoadyuvant) chemotherapy. Semin Oncol 1987; 14(1):1-17.

4. Systemic treatment of early breast cancer by hormonal, cytotoxic, or immune therapy: 133 randomised trials involving 31,000 recurrences and 24,000 deaths among 75,000 women. Early Breast Cancer Trialists' Collaborative Group. Lancet 1992; 339:1-15, 71-85.

5. Tamoxifen for early breast cancer: an overview of the randomised trials. Early Breast Cancer Trialists' Collaborative Group. Lancet 1998; 351:1451-1467.

6. Polychemotherapy for early breast cancer: an overview of randomised trials. Early Breast Cancer Trialists' Collaborative Group. Lancet 1998; 352:930-42.

7. Bonadonna G, Valagussa P, Moliterni A, et al. Adyuvant cyclophosphamida, methotrexate and fluoracil in node-positive brast cancer. The results of 20 years of follow-up. N Engl J Med 1995; 332: 901-6.

8. Castiglione-Gertsch $M$, Johnsen $C$, Goldhirsch A, et al. The Internacional (Ludwing) Breast Cancer Study Group Trials I-IV: 15 years of follow-up. Ann Oncol 1994; 5:717.

9. Goldhirsch A, Glick JH, Gelber RD, et al: Meeting highlights: International Consensus Panel on the Treatment of Primary Breast Cancer. J Clin Oncol 2001; 19:38173827.

10. Rosen PP, Groshe S, Kinne DW: Survival and prognostic factors in node-negative breast cancer: long-term follow-up studies. J Natl Cancer Inst Monogr 1992; 11:159-162.
11. Fisher B, Fisher ER, Redmond C, et al.: Tumor nuclear grade, estrogen receptor, and progesterone receptor: their value alone or in combination as indicators of outcome following adjuvant therapy for breast cancer. Breast Cancer Research and Treatment, 1986; 7(3):147160.

12. Fisher B, Dignam J, Mamounas EP, et al.: Sequential methotrexate and fluorouracil for the treatment of nodenegative breast cancer patients with estrogen receptornegative tumors: eight-year results from National Surgical Adjuvant Breast and Bowel Project (NSABP) B-13 and first report of findings from NSABP B-19 comparing methotrexate and fluorouracil with conventional cyclophosphamide, methotrexate, and fluorouracil. J Clin Oncol 1996; 14(7):1982-1992.

13. Mansour EG, Gray R, Shatila AH, et al.: Survival advantage of adjuvant chemotherapy in high-risk node-negative breast cancer: ten-year analysis: An Intergroup study. J Clin Oncol 1998; 16(11):3486-3492.

14. Zambetti M, Valagussa P, Bonnadonna G, Adyuvant cyclophosphamida, methotrexate and fluoracil in node negative and receptor negative brast cancer. Update results. Ann Oncol 1996; 7:481.

15. Adjuvant chemotherapy for breast cancer. National Institutes of Health Consensus Development Conference Statement. Natl Inst Health Consens Dev Conf Consens Statement 1985; 5:5 p.

16. Glick JH, Gelbert RD, Goldhirsch A, Senn HJ. Adjuvant therapy of primary breast cancer. 4th International Conference on Adjuvant Therapy of Primary Breast Cancer St. Gallen, Switzerland. Ann Oncol 1992 Dec; 3 (10):801-7.

17. Kaplan EL, Meier P. Nonparametric estimation from incomplete observations. J Am Statist Assoc 1958; 53, 457-481.

18. Mantel N. Evaluation of survival data and two new rank order statistic arising in its consideration. Cancer Chemother Rep 1966; 50, 163-167.

19. Hrynuik WM, Goodyear M. The calculation of received dose intensity. J Clin Oncol 1990; 8:1935-1937.

20. Fisher B, Dignam J, Bryant J, et al.: Five versus more than five years of tamoxifen for lymph node-negative breast cancer: updated findings from the National Surgical Adjuvant Breast and Bowel Project B-14 randomized trial. J Natl Cancer Inst 2001; 93 (9):684-90.

21. Fisher B, Anderson S, Tan-Chiu E, et al.: Tamoxifen and chemotherapy for axillary node-negative, estrogen receptor-negative breast cancer: findings from National Surgical Adjuvant Breast and Bowel Project B-23. J Clin Oncol 2001; 19(4):931-42.

22. Fisher B, Costantino JP, Redmond CK, et al.: Endometrial cancer in tamoxifen-treated breast cancer patients: findings from the National Surgical Adjuvant Breast and Bowel Project (NSABP) B-14. J Natl Cancer Inst 1994 86 (7):527-37.

23. Pritchard $\mathrm{KI}$, Paterson $\mathrm{AH}$, Paul NA, et al.: Increased th- 


\section{E. del Barco Morillo y cols.}

romboembolic complications with concurrent tamoxifen and chemotherapy in a randomized trial of adjuvant therapy for women with breast cancer. National Cancer Institute of Canada Clinical Trials Group Breast Cancer Site Group. J Clin Oncol 1996; 14 (10): 2731-7.

24. Albain KS, Green SJ, Ravdin PM et al. Adjuvant chemohormonal therapy for primary breast cancer should be sequential instead of concurrent; initial results from intergroup trial 0100 (SWOG-8814). Proc Am Soc Clin Oncol 2002; 21:37a.

25. Pico C, Martin M, Jara A et al. Epirubicin-cyclophosphamide (EC) chemotherapy plus tamoxifen (T) administered concurrent (con) versus sequential (sec): randomized phase III trial in postmenopausal node-positive breast cancer (BC) patients. Proc Am Soc Clin Oncol 2002; 2:37a.

26. Dignam JJ, Fisher B: Occurrence of stroke with tamoxifen in NSABP B-24. Lancet 2000; 355(9206): 848-849.

27. Cascinelli N, Greco M, Bufalino R, et al.: Prognosis of breast cancer with axillary node metastases after surgical treatment only. Euro J Cancer and Clin Oncol 1987; 23(6):795-799.

28. Fisher B, Redmond C, Wickerham DL, et al.: Systemic therapy in patients with node-negative breast cancer: a commentary based on two National Surgical Adjuvant Breast and Bowel Project (NSABP) clinical trials. Annals of Internal Medicine 1989; 111 (9):703-712.

29. Moliterni A, Bonadonna G, Valagussa P et al. Cyclophosphamide, methotrexate and fluoracil with and without doxorubucin in the adjuvant treatment of resecable breast cancer with one to three positive axillary nodes. J Clin Oncol 1991; 9:1124-30.

30. Hutchins L, Green S, Ravdin P, et al.: CMF versus CAF +/- tamoxifen in high-risk node-negative breast cancer patients and a natural history follow-up study in low-risk node-negative patients: update of tamoxifen results. Breast Cancer Res Treat 1999; 57(1):25.

31. Hortobagyi GN, Frye D, Buzdar AU et al. Decreased cardiac toxicity of doxorubicin administered by continuous intravenous infusion in combination chemotherapy for metastatic breast carcinoma. Cancer 1989; 63:37-45.

32. Diamandidou E, Buzdar AU, Smith TL et al. Treatment- related leukemia in breast cancer patients treated with fluorouracil-doxorubicin-cyclophosphamide combination adjuvant chemotherapy: the University of Texas M.D. Anderson Cancer Center experience. J Clin Oncol 1996; 14:2722-2730.

33. Eifel P, Axelson JA, Costa J et al. National Institutes of Health Consensus Development Conference Statement: adjuvant therapy for breast cancer, November 1-3, 2000. J Natl Cancer Inst 2001; (93):979-989.

34. Levine MN, Bramwell VH, Pritchard Kl, et al. Randomized trial of intensive cyclophosphamide, epirubicin, and fluorouracil chemotherapy compared with cyclophosphamide, methotrexate, and fluorouracil in premenopausal women with node-positive breast cancer. National Cancer Institute of Canada Clinical Trials Group. J Clin Oncol 1998; 16(8):2651-2658.

35. Budman DR, Berry DA, Cirrincione CT, et al.: Dose and dose intensity as determinants of outcome in the adjuvant treatment of breast cancer. The Cancer and Leukemia Group B. J Natl Cancer Inst 1998; 90 (16): 1205-11.

36. Bonadonna $G$ and Balagussa $P$. Dose-response effect of adjuvant chemotherapy in breast cancer. $N$ Engl Med 1981; 304: 10-15.

37. Wood W, Budman D, Korzum A. Dose and dose intensity of adjuvant chemotherapy for stage II, node-positive breast carcinoma. N Engl Med 1994; 330: 1253-1259.

38. Fisher B, Anderson S, Wickerham DL, et al.: Increased intensification and total dose of cyclophosphamide in a doxorubicin-cyclophosphamide regimen for the treatment of primary breast cancer: findings from National Surgical Adjuvant Breast and Bowel Project B-22. J Clin Oncol 1997; 15 (5):1858-69.

39. Fisher B, Anderson S, DeCillis A, et al.: Further evaluation of intensified and increased total dose of cyclophosphamide for the treatment of primary breast cancer: findings from National Surgical Adjuvant Breast and Bowel Project B-25. J Clin Oncol 1999; 17 (11):3374-88.

40. Farquhar C, Basser R, Marjoribanks J, Lethaby A. High dose chemotherapy and autologous bone marrow or stem cell transplantation versus conventional chemotherapy for women with early poor prognosis breast cancer (Cochrane Review). In: The Cochrane Library, Issue 2 2003. Oxford: Update Software. 